\title{
Physics Sensitivity Studies at Korean Neutrino Observatory
}

\author{
Sanghoon Jeon ${ }^{* a}$ and Sangyong $\mathrm{Kim}^{b}$ \\ ${ }^{a}$ Sungkyunkwan University \\ ${ }^{b}$ Seoul National University \\ E-mail: physicoon0607@gmail.com, sfc5302@gmail.com
}

\begin{abstract}
The Korean Neutrino Observatory (KNO) is proposed as a next generation underground neutrino detector in Korea. The detector is a 260 kiloton water Cherenkov detector and can serve as the second detector of Hyper-Kamiokande experiment. By detecting J- PARC neutrino beam in these two detectors in Korea and Japan at the same time, the measurement of neutrino oscillation parameters such as leptonic $\mathrm{CP}$ phase and the neutrino mass ordering is expected to be improved. Physics sensitivity studies are performed with improved handling of systematic uncertainties compared to the previous study. We present preliminary results on physics sensitivities in various configurations of Hyper-Kamiokande experiment including the KNO configuration.
\end{abstract}

EPS-HEP 2017, European Physical Society conference on High Energy Physics

5-12 July 2017

Venice, Italy

${ }^{*}$ Speaker. 


\section{Introduction}

The Hyper-Kamiokande (Hyper-K) detector is a next generation underground water Cherenkov detector with the total mass of $260 \mathrm{kton}$ which serve as a far detector for the J-PARK neutrino beam. The main purpose of the Hyper-K detector is to observe $\mathrm{CP}$ violation in the neutrino oscillations and to determine the neutrino mass hierarchy [1]. KNO is the 2nd detector proposed to enhance physics potentials of the Hyper-K experiment.

\section{Sensitivity Study}

The sensitivities are evaluated by generating pseudo data from Monte Carlo simulation [2]. The expected event rates of the pseudo data are estimated by using NEUT 5.3.2, neutrino interaction generator, and a GEANT3-based simulation of the Super-K detector, scaling the fiducial mass from 22.5 kton to 187 kton. 10 years operation with $1.3 \mathrm{MW}$ of a beam power and a 1:3 ratio of $v$ mode to $\bar{v}$ mode is assumed, which corresponds to $27 \times 10^{21}$ protons on target. Oscillation probabilities are calculated using Prob3++. For the pseudo data, true values of oscillation parameters from the Particle Data Group are used. A matter density of $2.7 \mathrm{~g} / \mathrm{cm}^{3}$ with the $295 \mathrm{~km}$ baseline and $2.5^{\circ}$ offaxis angle is assumed for Japan Detector (JD), and $3.0 \mathrm{~g} / \mathrm{cm}^{3}$ with $1088 \mathrm{~km}$ and $1.3^{\circ}$ is assumed for Mt.Bisul, which is one of the candidate sites of Korean Detector (KD) [3]. Simplified systematic uncertainties of Super-K are used in the sensitivity study.

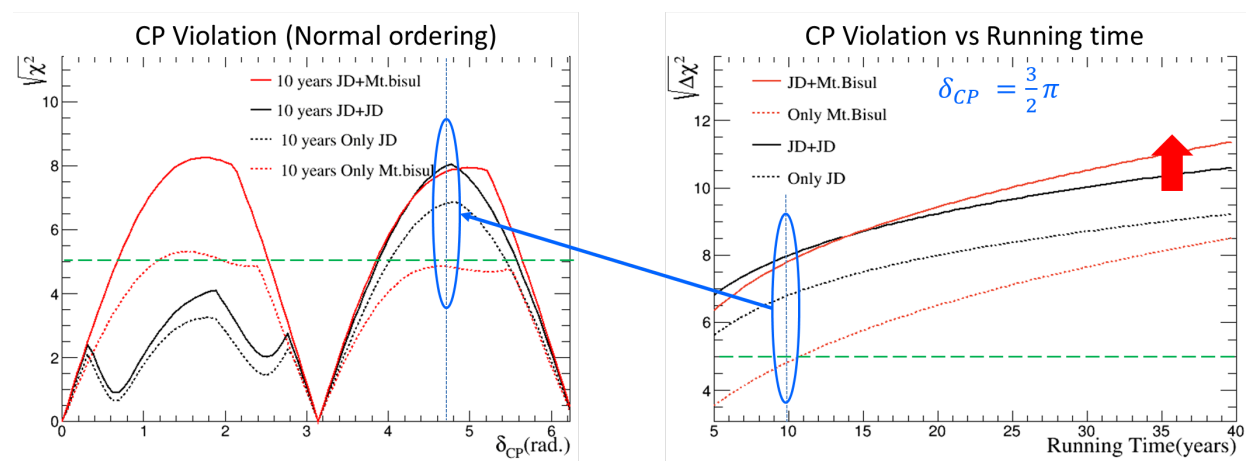

Figure 1: Sensitivity to $\mathrm{CP}$ violation : The left figure shows significance of $\mathrm{CP}$ violation measurement as a function of $\delta_{c p}$. The right figure shows the improvement of the significance over running time.

\section{Result}

As shown in Figuire 1, the KNO configuration in which the second detector is located at Mt.Bisul gives the best sensitivities for $\mathrm{CP}$ violation measurement.

\section{References}

[1] K. Abe et al, Hyper-K design report, arXiv:1805.04163v1 [physics.ins-det], 9 May 2018

[2] "T2HKKSensitivity-master", analysis package by M. Hartz

[3] K. Abe et al, (The Hyper-Kamiokande Proto-Collaboration), PTEP 2018, 6, 1-56 